\title{
Civilisations
}

Revue internationale d'anthropologie et de sciences

humaines

$54 \mid 2006$

Expériences de recherche en République

démocratique du Congo

\section{Les mesures de la dissimulation}

Les méthodes de recherche en sciences sociales face à la falsification de l'économie congolaise

\section{Benjamin Rubbers}

\section{(2) OpenEdition}

\section{Journals}

Édition électronique

URL : http://journals.openedition.org/civilisations/321

DOI : $10.4000 /$ civilisations. 321

ISSN : 2032-0442

Éditeur

Institut de sociologie de l'Université Libre de Bruxelles

\section{Édition imprimée}

Date de publication : 1 avril 2006

Pagination : $57-66$

ISBN : 2-87263-006-6

ISSN : 0009-8140

Référence électronique

Benjamin Rubbers, «Les mesures de la dissimulation », Civilisations [En ligne], 54 | 2006, mis en ligne le 01 avril 2009, consulté le 07 mai 2019. URL : http://journals.openedition.org/civilisations/321 ; DOI : 10.4000/civilisations.321 


\title{
Les mesures de la dissimulation \\ Les méthodes de recherche en sciences sociales face à la falsification de l'économie congolaise
}

\author{
Benjamin RUBBERS
}

Résumé : Cet article propose une réflexion sur les problèmes de méthode que rencontre un anthropologue dans l'étude des pratiques économiques en République Démocratique $d u$ Congo. Il se livre à un examen critique des contraintes que le contexte sociologique impose à l'emploi de différentes techniques de collecte de données : l'exploitation des sources documentaires, l'administration de questionnaires, l'observation et l'entretien. Ainsi est mis en évidence l'éclairage que chaque démarche est susceptible de fournir sur la vie matérielle au Congo. L'objectif est de faire ressortir, de manière aussi précise que possible, la contribution que la recherche en sciences sociales peut apporter à la compréhension de l'économie en Afrique.

Mots-clés : économie, falsification, questionnaire, observation, entretien, Lubumbashi.

Summary: This article addresses the methodological challenges facing an anthropologist studying economic practices in DRC. It critically examines the constraints that the sociological context imposes upon the use of different information gathering techniques (recourse to written documents, administration of questionnaires, observation and interviews). The implications of each approach on material conditions in the Congo are thus made clear. The objective is to highlight as precisely as possible, the contribution that social science research can have on understanding economic practices in Africa.

Key words: economy, falsification, questionnaire, observation, interview, Lubumbashi. 
$\mathrm{O}$ n assiste depuis trente ans à l'épuisement du modèle économique mercantiliste issu de la période coloniale. La baisse du cours des matières premières, l'accroissement de l'endettement public, la détérioration du tissu industriel et l'échec des mesures de l'ajustement structurel réduisent le contrôle du pouvoir central sur les ressources de l'extraversion (Young et Turner 1985). Se dessine alors, de manière progressive mais nette, une nouvelle séquence historique dont il reste encore à comprendre la portée (Bayart, Ellis et Hibou 1997). Elle se caractérise pour le moment par un éclatement des filières de commercialisation qui connectent l'économie locale au marché mondial, ainsi que par un effilochement du politique : le réseau clientéliste unique cède la place à une série de factions régionales en compétition pour la maîtrise des trafics transfrontaliers (Botte 2002). Dès lors, la société congolaise observe un écartèlement de plus en plus marqué entre la structure étatique officielle et une pluralité de circuits de l'ombre (Reno 1998).

Comment étudier les pratiques économiques dans cet univers institutionnel en voie de falsification? C'est la question à laquelle je tente de répondre dans cet article en évaluant de manière critique la pertinence des sources documentaires, de la démarche quantitative et de la méthode qualitative dans le contexte congolais. Je me base pour ce faire sur mon expérience de terrain depuis 1999 : je me suis notamment penché, au cours de cette période, sur les échanges entre ville et village ainsi que sur l'insertion professionnelle des diplômés de l'Université de Lubumbashi (Rubbers 2000, 2003). Mais je recours exclusivement dans ce texte à des illustrations venant de ma recherche doctorale sur le monde social du capitalisme au Katanga.

\section{Les ressources documentaires}

Divers organismes produisent des chiffres sur l'économie congolaise, mais ceuxci fournissent en général une image erronée de la réalité. C'est le cas des données quantitatives qui portent sur la circulation commerciale à la frontière. D'une part, il est possible de faire transiter les marchandises avec une fausse déclaration : la manière la plus classique de frauder consiste à modifier la nature, la quantité ou la valeur de la cargaison pour se partager l'équivalent du manque à gagner de l'administration publique (trop peu perçu) entre le marchand, le transitaire et l'agent en fonction. Les chiffres attestant de l'importation de 1000 tonnes de farine de froment peuvent ainsi dissimuler l'entrée de 2000 tonnes de ciment. D'autre part, un nombre considérable de petits marchands opèrent dans la clandestinité : ils emploient souvent des journaliers (moineaux) pour traverser la frontière avec la marchandise sur le dos (bilanga). Une partie du flux international échappe de la sorte, via la contrebande, à l'enregistrement documentaire de la douane. Dès lors, les informations officielles non seulement donnent une estimation inexacte du poids relatif des divers articles dans l'échange (farine, lait en poudre, ciment, etc.), mais encore sous-estiment l'importance générale du commerce en Afrique.

L'administration rassemble aussi des statistiques sur les entreprises établies sur le territoire national. La division provinciale du Ministère du Plan possède ainsi un fichier rassemblant diverses informations sur les entreprises de Lubumbashi. Mais la démarche pour obtenir ce document est fastidieuse, voire coûteuse, pour un résultat qui procure peu 
de satisfaction : une liste incomplète qui, tout en comptant des sociétés qui ont disparu depuis des années, ne reprend pas des établissements bien connus aujourd'hui. En outre, les données sur chaque entreprise sous-évaluent leur bénéfice, leur personnel ainsi que leur masse salariale. C'est que les patrons cherchent à réduire leur puissance financière dans les registres officiels tandis que les fonctionnaires ne perçoivent pas l'utilité de tenir le recensement à jour. De ce fait, les opérateurs économiques soudoient les agents du ministère pour fournir une fausse déclaration, voire disparaître complètement de l'index.

Malgré cela, les données quantitatives nous donnent parfois une idée sur l'ampleur des trafics irréguliers à partir de la comparaison des sources sur une même marchandise. En 1989, le journal The Consolidated Gold Field estime ainsi la production nationale en or à douze tonnes alors que le Centre National d'Expertise livre seulement le chiffre de trois tonnes (MacGaffey et Ellis 1996 : 23). De même, plusieurs auteurs nous apprennent que le marché clandestin du diamant congolais dépasse de loin la vente officielle : les chiffres de l'importation sur le marché européen ne correspondent pas à ceux de la production au Congo (Misser et Vallée 1999; Monnier, Jewsiewicki et de Villers 2000). Enfin, le Rwanda exporte des quantités importantes de coltan durant la guerre, alors que son soussol ne possède aucun dépôt de ce métal utilisé dans des applications de haute technologie (Cuvelier 2004). Mais ce genre de source devient très difficile à trouver depuis presque dix ans : les organismes de coopération internationale manquent de données pour remplir, dans leur répertoire, la case de la République Démocratique du Congo.

La fiabilité de ces chiffres dépend en outre de la possibilité de certifier l'origine de la marchandise. On peut affirmer sans trop de risque que le coltan acheté à des marchands africains en Belgique provient du Congo. Mais comment authentifier l'origine du cobalt écoulé en Afrique du Sud? Ce métal passe en fraude la frontière congolaise pour être mélangé, voire fondu, avec le cobalt de la Zambie, avant de poursuivre sa route vers Durban. La vraisemblance des statistiques sur le commerce est par ailleurs fonction de la trajectoire suivie par le produit sur le marché international. La trace du diamant est perdue, par exemple, quand il est exporté de manière clandestine à Dubaï puis revendu dans le circuit officiel en Inde. Enfin, la différence obtenue entre plusieurs sources de données ne correspond pas nécessairement à la contrebande, car elle peut procéder aussi de la manière dont chaque organisme élabore son corpus statistique : les recherches sur le commerce africain reposent rarement sur la même source, voire sur la même méthodologie (Hibou 1996 : 45-91). Au bout du compte, force est donc de constater que la confrontation des résultats statistiques offre, en général, un éclairage plutôt médiocre sur l'économie en Afrique.

La presse locale ne livre pour sa part aucune donnée factuelle digne de foi depuis que le journaliste vit moins de la vente du journal que des émoluments de la personne sur qui il écrit un article : il peut réaliser un reportage favorable sur un événement public pour recevoir une rétribution des organisateurs en contrepartie, comme il peut attaquer un homme de manière à lui réclamer une rançon contre la réhabilitation de son image dans une prochaine édition. Le document journalistique doit donc être ramené à la stratégie de survie de son auteur. Celle-ci consiste à construire une relation de réciprocité avec un mécène, quitte à la transmuer en chantage si nécessaire.

Mais l'article de presse se révèle profitable quand il est étudié comme une rumeur qui circule au sein de l'arène politique locale : il nous informe sur la nature des conflits, sur la position des protagonistes ainsi que sur le mode de transmission de l'information au sein de la société. De même, il constitue en tant que document de flatterie ou de 
blâme une illustration de la manière dont se fabrique la relation de pouvoir au Congo (Callaghy 1980; Mbembe et Roitman 1995; Mbembe 1999). Le texte peut être utilement examiné, enfin, sous la loupe de l'herméneutique, pour faire ressortir le lexique utilisé à propos des opérateurs économiques de la place : la manière dont on traite de leur réussite, de leur relation au personnel ou encore de leur rôle dans la région nous renseigne non seulement sur leur popularité mais aussi, de manière plus générale, sur l'économie morale du capitalisme en Afrique.

Voici par exemple un extrait du journal $L a$ Tribune à propos de la situation des travailleurs dans une entreprise de traitement métallurgique à Likasi : "Des hommes réduits aux dimensions incarcérales [sic] d'automates, couverts de siures [sic] de poussières noires dans une atmosphère emplie de vapeur de sulfate de sulfure et autre acide mortel, ces pauvres congolais, au nom de la survie, se sont résolus à sacrifier leur vie pour des impitoyables capitalistes qui s'engraissent de la sueur de leurs employés » (5 juin 2003 : 4). Cet article truffé de fautes orthographiques divulgue des informations inexactes: l'usine emploie par exemple un procédé à sec sans aucun produit chimique. Mais son intérêt se situe au niveau de la manière dont il rend compte de la relation salariale dans les termes de l'exploitation capitaliste : il récupère le lexique du tiers-mondisme pour donner forme à une théorie locale de la conspiration de l'Occident entre l'Afrique (Bruckner 1983). Ce thème du joug est en même temps associé au registre de la corruption qui est un ingrédient omniprésent dans le discours populaire sur la réussite : le patron de la société sait « manipuler tout le monde à coup de billets verts », est-il écrit plus loin sans aucune pièce à conviction (Blundo et Olivier de Sardan 2001).

Les documents que produisent les entreprises sont difficiles à obtenir car ils contiennent des informations sur un mode de fonctionnement que l'opérateur économique cherche généralement à dissimuler, pour éviter toute forme de chantage. Une pièce tel un contrat de fourniture livre pourtant des renseignements fiables sur la nature du partenariat, sur les modalités de la livraison ainsi que sur les stratégies de protection contre le risque. Ainsi, la minceur du dossier de même que l'inconsistance du texte cachent souvent un accord léonin : le flou est destiné à donner à une société une marge de manœuvre aussi large que possible pour manipuler son partenaire. C'est pourquoi les conventions que la société publique Gécamines signe avec un opérateur privé semblent à ce point sommaires : l'organe de direction est lui-même intéressé au bradage des actifs de l'entreprise étatique.

Restent enfin les actes des procès que j'ai pu obtenir auprès du greffier du tribunal de la ville: ils donnent un aperçu de la relation entre l'entrepreneur économique, l'administration, le personnel et ses partenaires (fournisseurs, concurrents, clients). Cette source nous documente sur des conflits que les acteurs tendent à cacher, sur le mode de justification juridique des pratiques économiques ou encore sur l'efficacité des mécanismes de répression. Mais le processus bureaucratique est ici, comme ailleurs, court-circuité par des sociabilités que l'argent alimente à l'occasion : le juge accepte parfois, contre un petit cadeau, de faire pencher la balance ${ }^{1}$. Dès lors, comment évaluer en toute sincérité le caractère établi ou non des faits repris dans le compte-rendu de la séance publique?

1. L'argent est un outil privilégié de production du lien social au Congo : il est utilisé pour créer une interdépendance personnelle autant sur le marché que dans le domaine de la parenté ou de l'amitié. La monnaie ne définit pas ici, comme en Europe, une sphère publique impersonnelle où règnerait l'intérêt, par opposition à une sphère intime où dominerait l'abnégation (Bloch et Parry 1989). 
On est constamment ballotté pour ce faire entre l'autorité de la justice et le soupçon de vénalité qui pèse en permanence sur toute administration publique.

En conclusion, l'examen des sources documentaires appelle une remise en question du lexique sociologique sur l'économie congolaise. Le concept le plus utilisé à ce propos est sans conteste celui d'économie seconde : il rassemble dans une catégorie unique les activités qui échappent au contrôle de l'Etat (MacGaffey 1991). Or, une grande partie du commerce non enregistré implique la collaboration des agents de l'administration (Roitman 1990). L'inscription administrative représente simplement une mise en scène que l'opérateur économique négocie avec le fonctionnaire, si bien que l'étiquette « informelle » entretient une relation lâche avec la réalité (Becker 1985). Dès lors, tout comme il se différencie de l'activité illégale, le commerce non enregistré ne se confond guère avec le trafic caché. Il est nécessaire, pour l'étude des pratiques économiques, de considérer le contrôle effectif sur le terrain, l'encodage documentaire, l'imposition fiscale, la régularisation juridique et la légitimation morale comme des opérations distinctes pouvant chacune faire l'objet de transactions.

\section{La démarche quantitative}

Faute de trouver des statistiques fiables auprès de l'administration, j'ai engagé un géographe congolais pour effectuer un recensement des entreprises de Lubumbashi : ce dernier a parcouru les rues de la ville avec un questionnaire qui reprend, outre la localisation, les principales caractéristiques de chaque société. Cette manière de faire prend bien entendu du temps, coûte de l'argent et repose, comme le registre officiel, sur la bonne foi de l'opérateur économique : elle fournit donc très probablement une image équivoque de l'importance de l'entreprise (chiffre, bénéfice, personnel, etc.). Mais elle livre en retour une énumération plus complète des établissements de la localité tout en offrant des résultats dont la probité est bien plus grande pour les données qualitatives comme l'ancienneté, le secteur ou la nationalité de l'actionnaire majoritaire. En outre, elle donne l'opportunité de corroborer les chiffres remis par le patron : mon collaborateur congolais interroge ensuite la sentinelle tandis que je repasse moi-même chez le responsable pour obtenir un entretien. Ainsi, outre son intérêt intrinsèque, cet inventaire m'a permis de construire un échantillon qualitatif fiable en ce qui concerne les opérateurs avec pignon sur rue : il épuise dans cette catégorie l'intégralité des cas de figure, que l'anthropologue choisisse la nationalité, le secteur ou l'ancienneté comme critère de sélection.

L'enquête par questionnaire me semble par contre peu pertinente pour étudier la carrière des entrepreneurs économiques de la région. L'échantillonnage est impossible à réaliser car la population de base demeure inconnue : elle ne correspond en tout cas pas au nombre des établissements car de nombreux marchands congolais opèrent à partir de leur domicile, voire sur le trottoir. L'administration du questionnaire représente en outre une démarche trop brutale pour nouer avec l'opérateur privé une relation de confiance. Or, celle-ci est déterminante dans une société où les activités de commerce jouent souvent avec la normativité de l'administration publique.

Cette technique est par ailleurs difficile à mettre en œuvre dans une société prise dans un processus de falsification institutionnelle, car il est impossible de forger une nomenclature pertinente lorsque le découpage officiel est fictif: la robustesse des catégories de la recherche statistique dépend de l'objectivation sociale préalable par la structure étatique (de Singly 1992 : 21-22). Au Congo, la classification de tel individu comme cadre de la fonction publique ne livre, par exemple, aucune information valable 
sur son niveau scolaire, et encore moins sur son niveau de rémunération. Ce genre de corrélation statistique est ici très lâche comparativement à une société bureaucratique comme la France (Desrosières et Thévenot 1988). La raison en est que le jeu social ne se dévoile pas au grand jour dans la société congolaise.

Enfin, cette démarche débouche sur une approche déterministe : elle cherche à isoler des propriétés afin de révéler des déterminants de l'action qui échappent à la conscience des individus (Lahire 1995, 2001). Or, l'ambition de ma recherche est davantage de mettre en évidence la subtilité du jeu social pour reconstruire la configuration relationnelle au sein de laquelle est inscrite la trajectoire entrepreneuriale (de Certeau 1980). Dans une société du caché, il est à mon sens utile, avant de dresser la cartographie statistique du monde des affaires, de cerner comment les acteurs se jouent du terrain, construisent leur ethos économique et définissent leur appartenance sociale (Rosental 1996).

\section{La méthode qualitative}

Les pratiques sociales du monde des entreprises ne se livrent pas facilement à l'observation directe. Les transactions économiques se mènent en général loin des regards indiscrets, à moins de donner lieu à un rituel public, comme par exemple lors de l'ouverture des enveloppes après un appel d'offre. Les pratiques de concussion s'enchâssent le plus souvent dans une relation de réciprocité de longue durée dont l'anthropologue ignore l'histoire (Blundo 2003). Enfin, ce dernier ne peut pas observer incognito les interactions dans l'établissement lui-même, car sa présence comme étranger ne laisse personne indifférent, même le patron : il est vite assimilé à un espion. Bref, il me semble difficile de mettre en place une observation continue des pratiques autant dans un univers politique en ombre et lumière que dans une situation sociale où règne une certaine méfiance (Rubbers 2004a).

En revanche, l'observation ne pose aucun problème dans un lieu public, telle une salle d'attente dans une usine, ou encore dans un espace de confiance sociale, comme dans un cercle de loisirs. Du quotidien émerge ainsi, de manière fortuite, des données éparses sur la relation des entrepreneurs avec leur personnel, l'administration, leur clientèle ou encore les usagers de la rue. Ces situations viennent alors enrichir, ou infirmer, le discours de la personne qui est soumise à l'observation. La gestuelle théâtrale de l'opérateur économique avec une autorité militaire vient de la sorte illustrer son propos quand il me déclare en a parte: «Tu vois, Benjamin, comme on doit faire la pute dans ce pays ». L'observation participante donne aussi l'opportunité de reconstruire les cercles de sociabilité des entrepreneurs et de comprendre comment se construisent les relations de commerce. Le jeu de la concurrence économique au sein du monde expatrié se retrouve ainsi dans la fréquentation des clubs sportifs, dans la mesure où personne ne souhaite se retrouver avec son adversaire dans un lieu de camaraderie. Enfin, l'immersion offre une occasion de capter les rumeurs pour saisir l'usage qui leur est réservé dans un monde économique sensible à la respectabilité sociale : le potin est une arme comme une autre pour fragiliser un rival en affaires.

L'entretien me semble être, avec l'observation, la méthode la plus utile pour mener une recherche sur l'économie en Afrique : la longueur de l'entrevue favorise la confidence, l'échange facilite l'appréhension fine des pratiques dans leur contexte social tandis que la libre élocution donne accès par les mots, les expressions et le ton du discours, à la sémiologie de l'action économique. L'échantillonnage consiste alors à suivre, de proche en proche, le réseau relationnel de chaque interlocuteur (snowball sampling). Cette 
technique offre l'avantage de dépasser l'organigramme de l'entreprise pour toucher tous les acteurs en relation avec le monde des affaires, quelle que soit leur position officielle. De plus, elle renforce la confiance de l'interviewé dans la mesure où l'anthropologue ne représente plus pour lui un inconnu. Ce procédé est poursuivi jusqu'à la saturation théorique des données : celle-ci est atteinte quand on estime avoir épuisé la diversité des cas de figure (Strauss 1992). Ainsi est mise en évidence non pas la fréquence des pratiques, mais la multiplicité des mécanismes de l'engendrement du social.

Cependant, la recherche sociologique par entretien glisse rapidement, si on ne fait pas attention, vers l'enquête policière, car le monde des affaires est propice au jeu des intrigues : l'anthropologue est volontiers pris à partie pour authentifier une accusation contre autrui. Or, ce dérapage méthodologique qui consiste à ne retenir des pratiques économiques que leur caractère légal me semble périlleuse : la loi ne constitue pas dans le contexte congolais un domaine bien délimité, mais une référence à l'autorité que le fonctionnaire utilise de manière situationnelle à une fin de chantage. Dès lors, la démarche juridique risque de confondre le licite et le légitime, et de consolider ainsi les inégalités sociales en place (Rubbers 2004b). L'entretien implique certes une double transaction objective et subjective qui modèle, voire biaise, le contenu de la biographie (Dubar 1994, 1996). D’une part, l'individu élabore une mise en intrigue de son passé en fonction des hypothèses qu'il se fait sur mon compte : en général, je suis considéré dans le monde des affaires comme un porte-parole de la Belgique (journaliste de gauche, étudiant naïf, bailleur de fond riche à souhait, etc.). D'autre part, chacun sélectionne au cours de l'entretien des bribes de son vécu pour fournir une image valorisante de Soi (Bourdieu 1986) : la grande corruption, les détournements de fonds comme la xénophobie concernent toujours les autres, jamais l'interlocuteur lui-même. Mais l'anthropologue ne se trouve pas pour autant acculé, me semble-t-il, à l'investigation policière : il dispose de diverses procédures pour contrôler ses interprétations de manière empirique.

La plausibilité du discours est testable par le recoupement des données : la réitération des entrevues permet de juger la cohérence des informations fournies par une même personne, tandis que la confrontation des sources (entretien, documents, observation) établit la convergence ou non des points de vue. La simple interrogation sur la raison qui pousse chacun à accepter une interview est de même une façon de cerner l'orientation du discours et partant, de mieux en comprendre la portée. Ainsi, si le but du marchand congolais est de recevoir une aide financière, il ne risque pas de me vanter les « coups » réussis au cours de sa carrière, comme il le faisait la veille avec un ami : il se présente plutôt comme un honnête commerçant sans histoire. Il apparait aussi préférable de coopérer avec un chercheur congolais pour interroger les personnes qui entretiennent plus aisément une relation de complicité avec un compatriote. C'est le cas, par exemple, des travailleurs dans une société qui appartient à un expatrié : ils penseraient que je suis un indicateur au service du patron. Enfin, je n'ai pas encore eu l'occasion de confronter mes interprétations à la critique du public congolais. Mais la restitution fait partie de mes projets : elle représente pour moi un solide gage de rigueur et une manière subtile de désamorcer la tension qui résulte de ce genre de recherche (Dubet 1987).

\section{Conclusion}

L'ambition éthique de la recherche consiste à présenter à la société congolaise un miroir qui reflète sa vie économique, de manière à lui donner les clés qui lui permettront de construire son avenir. Les sciences sociales adoptent généralement pour ce faire le 
registre de la dénonciation : elles utilisent le lexique juridique de la corruption, du pillage ou encore de la criminalisation. Or, cette position est à mon sens intenable quand est admise l'idée que l'anthropologue ne possède aucun ascendant moral pour juger autrui : il est censé comprendre la signification que les acteurs accordent à leur existence, pour leur fournir en retour une image aussi dense que possible de leur agir (Boltanski et Thévenot 1991). Dès lors, un retour vers l'idéal du relativisme éthique me semble nécessaire pour ne pas tomber dans l'écueil du développementalisme, auquel correspond depuis toujours une pensée raciste ${ }^{2}$.

Mais la théorie ainsi construite doit, dans tous les cas, apparaître non pas comme une libre herméneutique, mais comme un chantier empirique (Passeron 1991). Nous gagnons pour cela, comme le montre cet article, à recourir de manière complémentaire à l'ensemble des méthodes en sciences sociales : l'opposition du quantitatif au qualitatif, ou encore celle de la source écrite à la source orale, est per se complètement absurde. Mais en même temps, à partir de mon expérience de terrain, je pense que la démarche qualitative mérite une place de premier plan : elle représente sans doute le moins mauvais outil pour interroger les pratiques économiques dans une région où le double règne sur l'ensemble de l'appareil politique.

Cette exigence empirique est importante dans une discipline ethnographique en proie au narcissisme méthodologique, et partant à la divagation dans l'interprétation (Olivier de Sardan 2000). Mais elle semble aussi essentielle dans une région volontiers investie par la porte imaginaire que Joseph Conrad ouvre dans Au cœur des ténèbres (Dunn 2003). Ainsi, l'idée souvent admise selon laquelle l'économie africaine serait en voie de marginalisation apparaît comme un ultime avatar du thème du retour du continent à la sauvagerie (Bayart 1999 : 106-108). Or, la recherche de terrain vient ici briser cette fausse évidence. Si elle accuse un amenuisement global de sa richesse, la société congolaise est plus que jamais insérée dans l'économie du monde. Il suffit pour s'en convaincre de suivre les interminables ramifications du commerce des produits miniers comme le cobalt, le diamant ou le coltan.

2. Cette exigence professionnelle n'empêche personne de retirer sa casquette de chercheur pour émettre une opinion personnelle : l'étude du nazisme, par exemple, ne se confond pas avec son acceptation. 


\section{Références bibliographiques}

Bayart, Jean-François, Stefen Ellis et Béatrice Hibou, 1997. La Criminalisation de l'Etat en Afrique. Bruxelles : Complexe.

BAYART, Jean-François, 1999. «L'Afrique dans le monde : une histoire d'extraversion », Critique internationale, $\mathrm{n}^{\circ} 5$, automne, pp. 97-120.

Becker, Howard, 1985 [1963]. Outsiders. Etudes de sociologie de la déviance. Paris : Métailié.

Bloch, Maurice et Jonathan PARry (éds), 1989. Money and The Morality of Exchange. Cambridge : Cambridge University Press.

Blundo, Giorgio, 2003. " Décrire le caché, autour du cas de la corruption », in Giorgio Blundo et Jean-Pierre Olivier de Sardan (éds), Pratiques de la description, pp. 75-111. Paris : Ecole des hautes études en sciences sociales.

Blundo, Giorgio et Jean-Pierre Olivier de SARdan, 2001. « Sémiologie populaire de la corruption », Politique africaine, 83, pp. 98-114.

Boltanski, Luc et Laurent Thevenot, 1991. De la justification. Les économies de la grandeur. Paris : Gallimard.

Botтe, Roger, 2002. « Economies trafiquantes et mondialisation. La voie africaine vers le 'développement'? », Politique africaine, 88, pp. 131-150.

Bourdieu, Pierre, 1986. «L'illusion biographique », Actes de la recherche en sciences sociales, juin, pp. 6263.

Bruckner, Pascal, 1983. Le sanglot de l'homme blanc, Tiers-monde, culpabilité, haine de soi. Paris : Le Seuil.

Callaghy, Thomas, 1980. « State-Subject Communication in Zaire : Domination and the Concept of Domain Consensus », The Journal of Modern African Studies, 18 (3), pp. 469-492.

De Certeau, Michel, 1980. L'invention du quotidien. Arts de faire. Paris : 10/18.

DE SINGLY, François, 1992. L'enquête et ses méthodes : le questionnaire. Paris : Nathan.

Ceyssens, Rik, 1975. « Mutumbula. Mythe de l'opprimé », Cultures et développement, 7 (3-4), pp. 483-550.

Cuvelier, Jeroen, 2004. «Réseaux de l'ombre et configurations régionales : le cas du commerce du coltan en République Démocratique du Congo », Politique africaine, 93, pp. 82-92.

Desrosieres, Alain et Laurent Thevenot, 1988. Les catégories socio-professionnelles. Paris : La Découverte, collection 'Repères'.

Dubar, Claude

1994. « L'insertion comme articulation temporelle du biographique et du structurel », Revue française de sociologie, 15 (2), pp. 283-291.

1996. La socialisation : construction des identités sociales et professionnelles. Paris : A. Colin.

Dubet, François, 1987. La Galère : jeunes en survie. Paris : Fayard.

Dunn, Kevin, 2003. Imagining the Congo. The International Relations of Identity. New York: Palgrave Macmillan.

Нıвоu, Béatrice, 1996. L’Afrique est-elle protectionniste? Les chemins buissonniers de la libéralisation extérieure. Paris : Karthala.

LAHIRE, Bernard

1995. Tableaux de famille. Heurts et malheurs scolaires en milieux populaires. Paris : Le Seuil/ Gallimard.

2001. L'homme pluriel. Les ressorts de l'action. Paris : Nathan. 
MacGaffey, Janet (éd.), 1991. The Real Economy of Zaire. The Contribution of Smuggling and Other Unofficial Activities to National Wealth. Londres et Philadelphie : James Currey et University of Pennsylvania Press.

MacGaffey, Janet et Stephen ElLIs, 1996. « Research on Sub-Saharan Africa's Unrecorded International Trade : Some Methodological and Conceptual Problems », African Studies Review, 39 (2), pp. 19-41.

Mbembe, Achille et Janet Roitman, 1995. « Figures of the Subject in Times of Crisis », Public Culture, 5 (2).

MвемвE, Achille, 1999. De la postcolonie. Essai sur l'imagination politique en Afrique. Paris : Karthala.

Misser, François et Olivier VAllée, 1999. Les gemmocraties. L'économie politique du diamant africain. Paris : Desclée de Brouwer.

Monnier, Laurent, Bogumil Jewsiewicki et Gauthier DE ViLlers (éds), 2000. Chasse au diamant au Congo/Zaire. Paris-Tervuren : L'Harmattan-Institut africain.

Olivier de Sardan, Jean-Pierre, 2000. « Le 'je’ méthodologique. Implication et explicitation dans l'enquête de terrain », Revue française de sociologie, 41 (3), pp. 417-445.

PASSERON, Jean-Claude, 1991. Le raisonnement sociologique. L'espace non-popperien du raisonnement naturel. Paris : Nathan.

Reno, William, 1998. Warlord Politics and African States. Londres : Lynne Rienner Publishers.

Roitman, Janet, 1990. «The Politics of Informal Markets in Sub-Saharan Africa », The Journal of Modern African Studies, 28 (4), pp. 671-696.

Rosental, Paul-André, 1996. «Construire le 'macro' par le 'micro': Fredrik Barth et la microstoria », in Jacques Revel (éd.), Jeux d'échelles. De la micro-analyse à l'expérience, pp. 141-159. Paris : Gallimard, Le Seuil.

RuBBERs, Benjamin

2000. Entre ville et village. Identité et échange chez les Lamba du Katanga. Université libre de Bruxelles : mémoire de licence.

2003. Devenir médecin en République Démocratique du Congo. La trajectoire socioprofessionnelle des diplômés en médecine de l'Université de Lubumbashi. Paris-Tervuren : L'Harmattan-Institut africain.

2004a. « Conversation sur la couleur des hommes », Politique, 35, pp. 43-45.

2004b. « La dislocation du secteur minier au Katanga. Pillage ou recomposition? », Politique africaine, 93, pp. 21-42.

Strauss, Anselm, 1992. La trame de la négociation. Sociologie qualitative et interactionnisme. Paris: l'Harmattan.

VelLut, Jean-Luc, 1982. « Matériaux pour une image du blanc dans la société coloniale du Congo belge », in Jean Pirotte (éd.), Stéréotypes nationaux et préjugés raciaux aux $19^{e}$ et $20^{e}$ siècles. Sources et méthodes pour une approche historique, pp. 91-116. Leuven : Editions Nauwelaerts.

Young, Crawford et Thomas Turner, 1985. The Rise and Decline of the Zairian State. Madison : University of Wisconsin Press. 


\title{
Un terrain fragmenté \\ Le kimbanguisme et ses ramifications
}

\author{
Anne MELICE
}

Résumé : Cet article considère mon expérience de terrain sur les sites kimbanguistes au Congo et en Belgique depuis 1996. Quelques écueils relatifs à ma position personnelle et à sa réceptivité par les Kimbanguistes sont évoqués d'entrée de jeu. Ensuite, je pointe les difficultés méthodologiques résultant de la diversification des sites kimbanguistes. Le concept d'ethnoscape que l'on doit à Arjun Appadurai permet d'interroger les processus et les modes de (re)configurations identitaires de groupes dispersés sur la planète. J'en viens, enfin, à des déplacements au-delà des sites kimbanguistes, en somme aux articulations de ces sites à d'autres sites non kimbanguistes. L'ethnographie multi-située et la métaphore du "pister » théorisées par George Marcus apportent un éclairage neuf sur la spécificité que présente ce terrain de recherche.

Mots-clés: kimbanguisme, ethnoscape, ethnographie multi-située, métaphore du « pister » .

Summary: This article is based on field work in Kimbanguist research sites in Congo and Belgium since 1996. Obstacles relating to my personal position and its receptivity by Kimbanguists are discussed at the outset. Next, I focus on the methodological difficulties associated with the diversity of Kimbanguist sites. The concept of ethnoscape developed by Arjun Appadurai helps us reflect on the processes and modes of identityrelated (re)configurations of groups dispersed all over the planet. The article ends with a discussion about displacements beyond the Kimbanguist sites. Multi-sited ethnography and the metaphor of 'tracking' theorised by George Marcus throws new light on the specificity of this research.

Key words: Kimbanguism, ethnoscape, multi-sited ethnography, tracking. 